\title{
Asymptotics of a Gauss hypergeometric function with two large parameters: a new case
}

\author{
J. F. Harper ${ }^{1}$
}

November 5, 2019

\begin{abstract}
Asymptotic expansions of the Gauss hypergeometric function with large parameters, $F\left(\alpha+\epsilon_{1} \tau, \beta+\epsilon_{2} \tau ; \gamma+\epsilon_{3} \tau ; z\right)$ as $|\tau| \rightarrow \infty$, are known for many special cases, but not for one that the author encountered in recent work on fluid mechanics: $\epsilon_{2}=0$ and $\epsilon_{3}=\epsilon_{1} z$. This paper gives the leading term for that case if $\beta$ is not a negative integer and $z$ is not on the branch cut $[1, \infty)$, and it shows how subsequent terms can be found.
\end{abstract}

\section{Introduction}

Asymptotic expansions as $|\tau| \rightarrow \infty$ of the hypergeometric function

$$
F\left(\alpha+\epsilon_{1} \tau, \beta+\epsilon_{2} \tau ; \gamma+\epsilon_{3} \tau ; z\right),
$$

where $\alpha, \beta, \gamma, \epsilon_{i}$ and $z$ are finite, have been studied for over 100 years but the theory is still not complete $[2,5,6,7,8]$. Most recently Paris [6] and then Cvitković et al. [2] considered $\varepsilon_{2}=0$ but their methods did not apply if $\varepsilon_{3}=\varepsilon_{1} z$. This paper deals with that case by putting $a=\alpha-\gamma / z, b=\beta$, $\lambda=\gamma+\epsilon_{3} \tau$, so that unless $z=0,|\lambda| \rightarrow \infty$ and

$$
F\left(\alpha+\epsilon_{1} \tau, \beta ; \gamma+\epsilon_{3} \tau ; z\right)=F(a+\lambda / z, b ; \lambda ; z) .
$$

We refer to this function simply as $F$ if no ambiguity would arise. If $z=0$, then (1) gives $F=1$; its asymptotic expansion is just 1 .

Our theory will assume that $b$ is not a negative integer, so that there is a branch cut in the complex $z$-plane, and that $z \notin[1, \infty)$ in order to avoid 
it. If $b$ were a negative integer, $F$ would be a polynomial in $z$ and a rational function of $\lambda$, no branch cut would exist, and the asymptotic series would converge if $|\lambda|>|b|-1$.

The motivation for studying the case $\varepsilon_{2}=0, \varepsilon_{3}=\varepsilon_{1} z$ was the author's recent theory [3] for a gas bubble rising in a solution of a substance (e.g. common salt in water) that raises the surface tension. Finding the drag on the bubble led to a series $\sum f_{n} \sigma_{n}$, in which $\left|f_{n}\right| \rightarrow 0$ as $n \rightarrow \infty$, and the values of $\sigma_{n}$ were

$$
\sigma_{n}=\frac{F\left(3 c_{n}+2,1 ; 2 c_{n}+2 ; \frac{2}{3}\right)}{1+2 c_{n}}-\frac{F\left(3 c_{n}+3,1 ; 2 c_{n}+3 ; \frac{2}{3}\right)}{1+c_{n}},
$$

where $c_{n} \rightarrow \infty$ as $n \rightarrow \infty$, and every $c_{n}>0$. Equation (3) involves two special cases of (2): $a=-\frac{1}{2} d, b=1, z=\frac{2}{3}, \lambda=2 c_{n}+d$, where $d=2$ or 3 . Section 3 will show that $\left|\sigma_{n}\right|=O\left(c_{n}^{-1 / 2}\right)$; the series $\sum f_{n} \sigma_{n}$ converges.

\section{Integral representation of $\boldsymbol{F}$}

Let ph $: \mathbb{C} \backslash\{0\} \rightarrow(-\pi, \pi]$ be the single-valued phase function $[5, \S 1.9(\mathrm{i})]$, let $\theta_{\lambda}=\operatorname{ph}(\lambda), \theta_{z}=\operatorname{ph}(z)$, let $a, b$ and $z$ be finite real or complex constants and let $G(\lambda, b), I(\lambda, a, b, z), p(t, z), q(t, a, b, z), r\left(t, z, \theta_{\lambda}\right)$ be the functions

$$
\begin{aligned}
G(\lambda, b) & =\frac{\Gamma(\lambda)}{\Gamma(b) \Gamma(\lambda-b)}, \\
I(\lambda, a, b, z) & =\int_{0}^{1} t^{b-1}(1-t)^{\lambda-b-1}(1-z t)^{-(\lambda / z)-a} d t \\
& =\int_{0}^{1} \exp (-\lambda p(t, z)) q(t, a, b, z) d t, \\
p(t, z) & =z^{-1} \ln (1-z t)-\ln (1-t), \\
q(t, a, b, z) & =t^{b-1}(1-t)^{-b-1}(1-z t)^{-a}, \\
r\left(t, z, \theta_{\lambda}\right) & =\operatorname{Re}\left(e^{i \theta_{\lambda}} p(t, z)\right) .
\end{aligned}
$$

If $z \neq 0$ or $1,|\operatorname{ph}(1-z)|<\pi$, and $\operatorname{Re}(\lambda)>\operatorname{Re}(b)>0$, then $[5, \S 15.6]$

$$
F(a+\lambda / z, b ; \lambda ; z)=G(\lambda, b) I(\lambda, a, b, z) .
$$


The asymptotic form of $G(\lambda, b)$ is well known $[5, \S 5.11($ iii) $]$. If $|\lambda| \rightarrow \infty$ and $\left|\theta_{\lambda}\right| \leqslant \pi-\delta<\pi$, then

$$
G(\lambda, b) \sim \frac{\lambda^{b}}{\Gamma(b)} \sum_{s=0}^{\infty}\left(\begin{array}{l}
b \\
s
\end{array}\right) \frac{B_{s}^{(b+1)}}{\lambda^{s}}=\frac{\lambda^{b}}{\Gamma(b)} \sum_{s=0}^{\infty} \frac{G_{s}}{\lambda^{s}}, \text { say }
$$

where the $B_{s}^{(b+1)}$ are Bernoulli numbers of order $b+1[5, \S 24.16(\mathrm{i})]$ :

$$
\begin{aligned}
& G_{0}=1, \\
& G_{1}=-b(b+1) / 2, \\
& G_{2}=b(b-1)(b+1)(3 b+2) / 24 .
\end{aligned}
$$

\section{Real $z<1, z \neq 0$}

Because we use both (6) and (10) we assume henceforth that $\left|\theta_{\lambda}\right|<\frac{1}{2} \pi, z \neq 0$, and $z \notin[1, \infty)$. We also assume for the time being that $\operatorname{Re}(\lambda)>\operatorname{Re}(b)>0$ and $z<1$, but the restriction on $\operatorname{Re}(b)$ will be relaxed in Section 3.2 and the restriction on $z$ will be relaxed in Section 4 .

\section{$3.1 \quad \operatorname{Re}(b)>0$}

Let $S$ be the open sector $\left\{t: 0<|t|<1,|\operatorname{ph}(t)|<\frac{1}{2} \pi\right\}$. Let $D$ be the open $\operatorname{disc}\{t:|t|<\min (1,1 /|z|)\}$. Let $A$ be the closed annular sector

$$
A=\left\{\lambda:|\lambda| \geqslant R_{\min }>\max (|b|+1,|z|+|a|),\left|\theta_{\lambda}\right| \leqslant \frac{1}{2} \pi-\delta<\frac{1}{2} \pi\right\} .
$$

We may use Laplace's method $[5, \S 2.4$ (iii)] if $z \in(-\infty, 0) \cup(0,1)$ to find the asymptotic series of $I(\lambda, a, b, z)$ by integrating along $P_{1}=[0,1]$ because the following conditions are all satisfied.

(i) Both $p(t, z)$ and $q(t, a, b, z)$ are analytic for $t \in S$, and the path of integration with $t$ real, from 0 to 1 , is in $S$ except for its ends.

(ii) If $t \in D$ then $p(t, z)$ and $q(t, a, b, z)$ have these convergent series in powers of $t$, with $p_{0} \neq 0$ and $q_{0} \neq 0$ :

$$
\begin{aligned}
p(t, z) & =\sum_{n=0}^{\infty} p_{n} t^{n+2}, \quad p_{n}=\frac{1-z^{n+1}}{n+2} \\
q(t, a, b, z) & =\sum_{n=0}^{\infty} q_{n} t^{n+b-1}, \quad q_{n}=(-1)^{n} \sum_{m=0}^{n}\left(\begin{array}{c}
-b-1 \\
n-m
\end{array}\right)\left(\begin{array}{c}
-a \\
m
\end{array}\right) z^{m} .
\end{aligned}
$$


If $b$ is not an integer we use the principal value of $t^{b}$ in (17).

(iii) $I(\lambda, a, b, z)$ converges at $t=1$ absolutely and uniformly with respect to $\lambda \in A$.

(iv) If $0<t<1$ then $r\left(t, z, \theta_{\lambda}\right)>r\left(0, z, \theta_{\lambda}\right)=0$.

(v) If $\lambda \in A, r\left(t, z, \theta_{\lambda}\right)$ is bounded away from zero uniformly with respect to $\theta_{\lambda}$ as $t \rightarrow 1$ along $P_{1}$.

Then, as $\lambda \rightarrow \infty$ in $S$,

$$
I(\lambda, a, b, z) \sim \sum_{s=0}^{\infty} \Gamma\left(\frac{s+b}{2}\right) \frac{a_{s}}{\lambda^{(s+b) / 2}},
$$

where the coefficient $a_{s}$ is the residue at $t=0$ of

$$
\frac{q(t, a, b, z)}{2 p(t, z)^{(s+b) / 2}},
$$

which has a pole of order $s$ there. Expressions for $a_{s}$ are also known in terms of partial ordinary Bell polynomials $[9,10]$ and of ordinary potential polynomials [4]. We have

$$
\begin{aligned}
& a_{0}=\frac{q_{0}}{2 p_{0}^{b / 2}}=\frac{2^{b / 2-1}}{(1-z)^{b / 2}}, \\
& a_{1}=\left[\frac{q_{1}}{2}-\frac{(b+1) p_{1} q_{0}}{4 p_{0}}\right] \frac{1}{p_{0}^{(b+1) / 2}}, \\
& a_{2}=\left[\frac{q_{2}}{2}-\frac{(b+2) p_{1} q_{1}}{4 p_{0}}+\left\{(b+4) p_{1}^{2}-4 p_{0} p_{2}\right\} \frac{(b+2) q_{0}}{16 p_{0}^{2}}\right] \frac{1}{p_{0}^{(b+2) / 2}} .
\end{aligned}
$$

By the duplication formula for gamma functions $[5,(5.5 .5)]$, the leading term when $z$ is real is

$$
F\left(a+\frac{\lambda}{z}, b ; \lambda ; z\right) \sim \frac{2^{-b / 2} \pi^{1 / 2} \lambda^{b / 2}}{(1-z)^{b / 2} \Gamma\left(\frac{1}{2}+b / 2\right)} .
$$




\section{$3.2 \operatorname{Re}(b) \leqslant 0$}

Equation (23) has been proved for $\operatorname{Re}(b)>0$. It still holds if $b=0$, because then $F=1$. It also holds for any $b \in \mathbb{C}$ except a negative integer. That is because the value for $\operatorname{Re}(b)>-1$ follows from those for $b+1$ and $b+2[1$, (15.2.11]. It agrees with (23), and shows that each term after the first in the asymptotic series is $O\left(\lambda^{-1 / 2}\right)$ times the previous one. In the same way we find the same results for $\operatorname{Re}(b)>-2,-3,-4, \ldots$ If on the other hand $b$ is a negative integer, the right-hand side of (23) is zero but the left-hand side is not. The asymptotic expansion then begins further along the series, which now converges and contains only integer powers of $\lambda$.

\section{Complex $z$}

We still require $\theta_{\lambda} \in\left(-\frac{1}{2} \pi, \frac{1}{2} \pi\right), z \neq 0$, and $z \notin[1, \infty)$. Checking where $r\left(t, z, \theta_{\lambda}\right)>0$ when $z \in \mathbb{C}$ need not require dealing with $\operatorname{Im}(z)<0$ because $r\left(t, z, \theta_{\lambda}\right)=r\left(t, \bar{z},-\theta_{\lambda}\right)$. If the least value of $r\left(t, z, \theta_{\lambda}\right)$ for $t \in P_{1}=[0,1]$ is still at $t=0$ even when $\operatorname{Im}(z) \neq 0$, then the result of Section 3 still holds.

We now show that a sufficient condition is $\left.\ddot{r}\left(t, z, \theta_{\lambda}\right)\right|_{t=0}>0$, where dots indicate $t$-derivatives. With $\theta_{\lambda}$ and $z$ fixed, $r\left(t, z, \theta_{\lambda}\right)$ defined in (9) is a real function of $t$ infinitely differentiable on $P_{1}$; its least value on $P_{1}$ is thus either at $t=0$ or at another point $t=t^{\prime}$ where $\dot{r}\left(t, z, \theta_{\lambda}\right)=0$. That gives a quadratic equation for $t$; its two roots are 0 and $t^{\prime}$. Because $r\left(t, z, \theta_{\lambda}\right) \rightarrow+\infty$ as $t \rightarrow 1$ along $P_{1}, t^{\prime} \in(0,1)$ would require $r\left(t^{\prime}, z, \theta_{\lambda}\right)<0$ and $\dot{r}\left(t^{\prime}, z, \theta_{\lambda}\right)=0$. Now

$$
\begin{aligned}
\dot{r}\left(t, z, \theta_{\lambda}\right) & =\operatorname{Re}\left(\frac{e^{i \theta_{\lambda}}}{1-t}-\frac{e^{i \theta_{\lambda}}}{1-z t}\right), \\
\therefore t^{\prime} & =\frac{\ddot{r}\left(0, z, \theta_{\lambda}\right)}{|z| \cos \left(\theta_{\lambda}-\theta_{z}\right)-|z|^{2} \cos \theta_{\lambda}}, \\
\ddot{r}\left(t, z, \theta_{\lambda}\right) & =\operatorname{Re}\left(\frac{e^{i \theta_{\lambda}}(1-z)\left(1-z t^{2}\right)}{(1-t)^{2}(1-z t)^{2}}\right) .
\end{aligned}
$$

If $\ddot{r}\left(0, z, \theta_{\lambda}\right)>0$ then $r\left(t, z, \theta_{\lambda}\right)>0$ when $0<t \ll 1$, and there would have to be a maximum of $r$ at $t^{\prime \prime} \in\left(0, t^{\prime}\right)$ before $r$ decreased to its minimum at $t^{\prime}$. The quadratic equation would then have three roots, which is impossible. Therefore if $\ddot{r}\left(0, z, \theta_{\lambda}\right)>0$ then $t^{\prime} \notin(0,1)$, and the leading contribution to the integral (6) is from $t$ near 0 , with $P_{1}$ still the path of integration. 


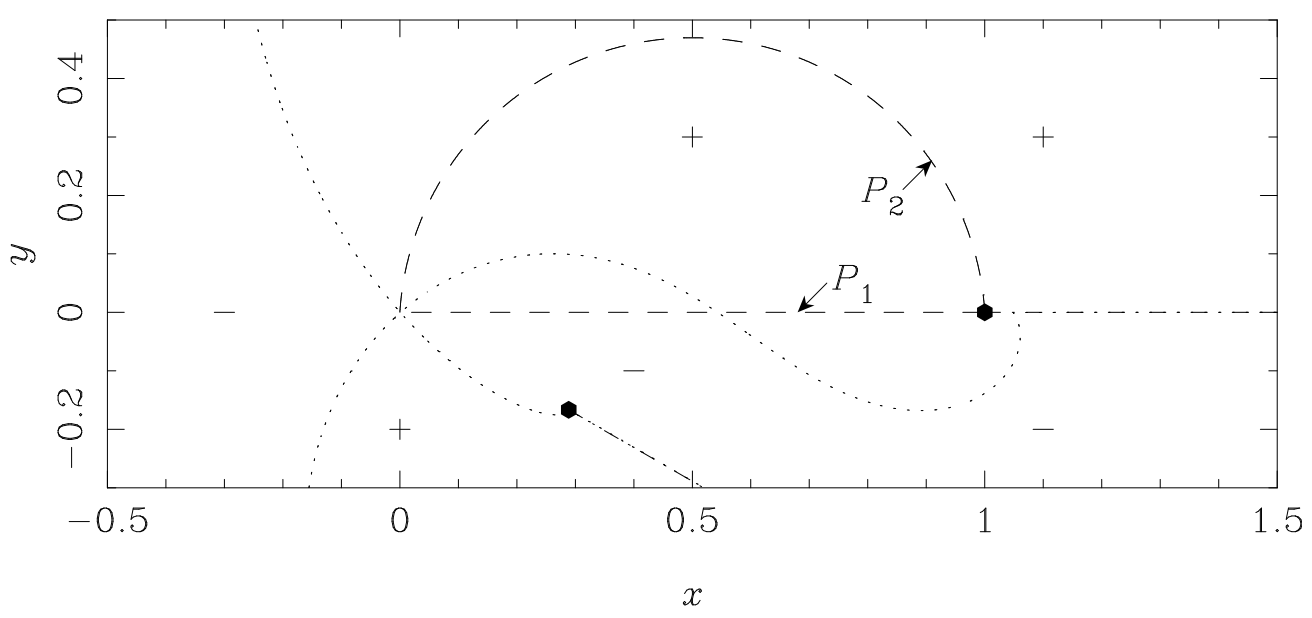

Figure 1: The complex $t$-plane $(t=x+i y)$, in a case where $P_{2}$ is useful but $P_{1}$ is not: $|z|=3, \theta_{z}=30^{\circ}, \theta_{\lambda}=-36^{\circ}$. Dotted lines on which $r\left(t, z, \theta_{\lambda}\right)=0$ separate regions where it is of constant sign, marked + and - . The lines spiral in towards the branch points on other Riemann sheets; only the principal sheet is shown. Dashed lines: the straight line $P_{1}$ and arc $P_{2}$. Dot-dash-dotdash lines: the branch cuts for $\ln (1-z t) / z$ and $\ln (1-t)$. Black circles: the branch points $t=1$ and $1 / z$.

Experiments with Waterloo Maple computer algebra revealed (to the author's initial surprise) that the results of Section 3 still held even when $\ddot{r}\left(0, z, \theta_{\lambda}\right)<0$. That is because there are other paths through complex values from $t=0$ to $t=1$ along which $r\left(t, z, \theta_{\lambda}\right)$ has its least value at $t=0$. Figures 1 and 2 illustrate the possibilities. In Figure $1|z|=3, \theta_{z}=30^{\circ}$, $\theta_{\lambda}=-36^{\circ}, \omega \approx 86^{\circ}$, and in Figure $2|z|=8, \theta_{z}=3^{\circ}, \theta_{\lambda}=83^{\circ}, \omega \approx 47^{\circ}$, where $\omega=-\frac{1}{2}\left\{\mathrm{ph}(1-z)+\theta_{\lambda}\right\}$.

Let $\theta_{t}=\operatorname{ph}(t)$. Then

$$
\begin{aligned}
r\left(t, z, \theta_{\lambda}\right) & \sim \operatorname{Re}\left(\frac{1}{2}(1-z) t^{2} e^{i \theta_{\lambda}}\right) \text { as } t \rightarrow 0 \\
& =\left|\frac{1}{2}(1-z) t^{2}\right| \cos \left(2 \theta_{t}-2 \omega\right) .
\end{aligned}
$$

Therefore $r\left(t, z, \theta_{\lambda}\right)$ begins increasing as $t$ leaves 0 if $\left|\theta_{t}-\omega\right|<\frac{1}{4} \pi$, and it does so as fast as possible if $\theta_{t}=\omega$. The other direction of steepest ascent of $r$ (or steepest descent of $e^{-\lambda p}$ ) from $t=0$ is $\theta_{t}=\omega \pm \pi$, but it will not concern us. Let $P_{2}$ be the circular arc passing through $t=0$ and $t=1$ with 


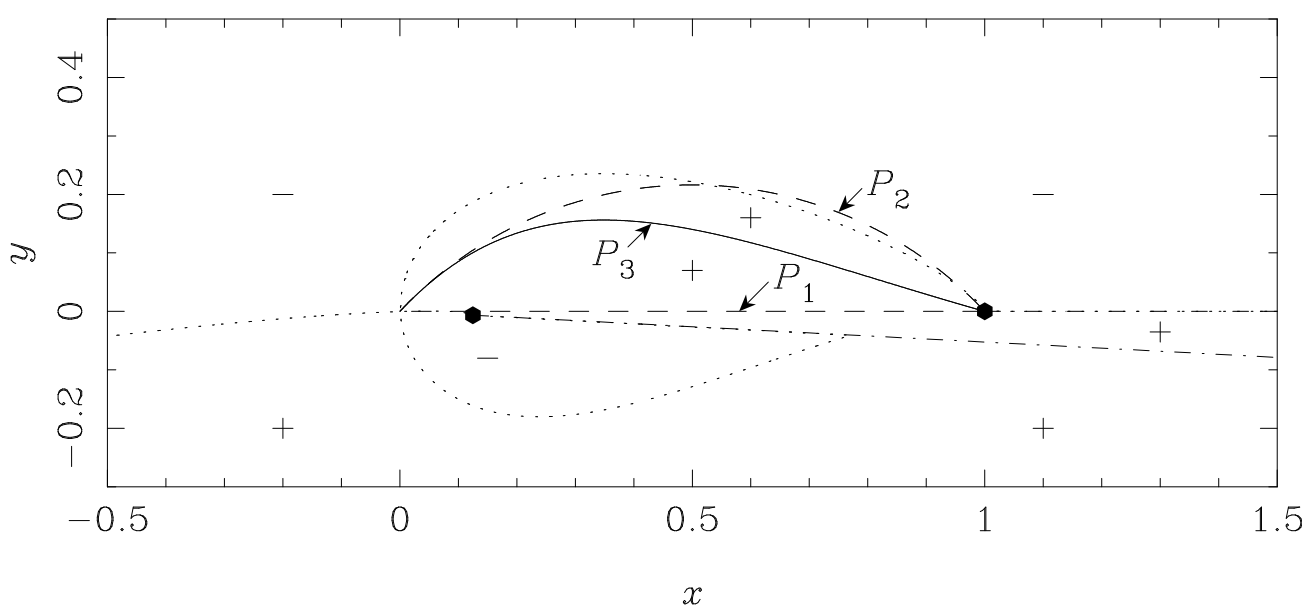

Figure 2: The complex $t$-plane in a case where $P_{3}$ is useful but $P_{1}$ and $P_{2}$ are not: $|z|=8, \theta_{z}=3^{\circ}, \theta_{\lambda}=83^{\circ}$. Solid line: $P_{3}$. Other symbols as in Figure 1.

its tangent at 0 in the direction $\theta_{t}=\omega$, so that if $\sin \omega \neq 0$

$$
t=\frac{(\sin \phi+\sin \omega)+i(\cos \phi-\cos \omega)}{2 \sin \omega}, \quad \phi \in[-\omega, \omega]
$$

Numerical work with $|z|$ from 0.5 to $10, \theta_{z}$ from $3^{\circ}$ to $177^{\circ}$, and $\theta_{\lambda}$ from $-87^{\circ}$ to $+87^{\circ}$ showed that $r\left(t, z, \theta_{\lambda}\right)>0$ everywhere except $t=0$ on at least one of $P_{1}$ and $P_{2}$, except for a few cases where $r\left(t, z, \theta_{\lambda}\right)<0$ at some points on each of $P_{1}$ and $P_{2}$. In those cases there is a path $P_{3}$ on which $r\left(t, z, \theta_{\lambda}\right)>0$ everywhere except $t=0$; we obtain $P_{3}$ by modifying $P_{2}$ to bring $y=\operatorname{Im}(t)$ nearer to 0 by an amount gradually increasing as $\phi$ increases:

$$
y=\frac{\cos \phi-\cos \omega}{2 \cosh (1+\phi / \omega) \sin \omega}, \quad \phi \in[-\omega, \omega]
$$

Because $t=0$ when $\phi=-\omega$ and $t=1$ when $\phi=\omega, P_{3}$ leaves $t=0$ in the same direction as $P_{2}$ but arrives at $t=1$ from a direction much closer to the real axis $y=0$; see Figure 2. $P_{3}$ was needed if $|z| \gg 1,\left|\theta_{z}\right| \ll \frac{1}{2} \pi$, so that $z$ was near the positive real axis but far from 1 , and $\theta_{\lambda}$ was near $\pm \frac{1}{2} \pi$. Then $r<0$ both on the straight line $P_{1}$ very near $t=0$ and on much of the $\operatorname{arc} P_{2}$; hence both paths were unsuitable for Laplace's method. Because $\omega \approx 47^{\circ}$ in Figure 2 , the lower right negative region is between $\theta_{t} \approx+2^{\circ}$ and $\theta_{t} \approx-88^{\circ}$ 
near $t=0$, and the path $P_{1}$ is initially inside it. The circular-arc path $P_{2}$ is in a negative region if $x>0.53$, but $P_{3}$ is in a positive region for its whole length. Note that $P_{2}$ and $P_{3}$ are not paths of steepest descent though they do leave $t=0$ in the steepest-descent direction. As the NIST Handbook says $[5, \S 2.4(\mathrm{iv})]$, "for the purpose of simply deriving the asymptotic expansions the use of steepest descent paths is not essential".

The remaining task was to choose the correct branch of $p_{0}^{b / 2}$ in (23). That was the one on which $\operatorname{ph}\left(p_{0}\right)=\operatorname{ph}(1-z)$ [5, eq. 2.4.13], whether the path of integration was $P_{1}, P_{2}$ or $P_{3}$.

\section{Conclusion}

The asymptotic form (23) for $F(a+\lambda / z, b ; \lambda ; z)$ as $|\lambda| \rightarrow \infty$ if $|\operatorname{ph} \lambda|<\frac{1}{2} \pi$ was found in Section 3.1 for $\operatorname{Re}(b)>0, z$ real, $z \neq 0$, and $z<1$. Section 3.2 shows that it still holds for any real or complex value of $b$ except a negative integer if $z \in \mathbb{R} \backslash[1, \infty)$, and Section 4 extends that region to $z \in \mathbb{C} \backslash[1, \infty)$. In a recent paper on bubbles rising in a liquid [3], $b=1$ and $z=\frac{2}{3}$, a special case which revealed that the present investigation was needed. The present results appear not to be in previous work on hypergeometric functions.

\section{Acknowledgements}

I wish to thank the authorities of the Victoria University of Wellington for allowing me to go on using its facilities after retirement. Graeme Wake, a friend and colleague for many years, might be surprised to know that his departure from that university helped me write this paper, because I then began teaching a course that included asymptotic expansions. It is notorious that teaching something is a good way to ensure that one learns it. I also wish to thank two referees; their suggestions improved this paper.

\section{References}

[1] Milton Abramowitz and Irene A. Stegun. Handbook of Mathematical Functions. Dover, 1972 (cit. on p. 5). 
[2] Mlslav Cvitković, Ana-Sunčara Smith, and Jayant Pande.

"Asymptotic expansions of the hypergeometric function with two large parameters - application to the partition function of a lattice gas in a field of traps". In: J. Phys. A: Math. Theor. 50 (2017). (24pp), p. 265206 (cit. on p. 1).

[3] J. F. Harper. "Effect of a negatively surface-active solute on a bubble rising in a liquid". In: Q. J. Mech. Appl. Maths 71 (2018), pp. 427-439. DOI: doi.org/10.1093/qjmam/hby012 (cit. on pp. 2, 8).

[4] Gergő Nemes. "An Explicit Formula for the Coefficients in Laplace's Method". In: Constr. Approx. 38 (2013), pp. 471-487. DOI: 10.1007/s00365-013-9202-6 (cit. on p. 4).

[5] Frank W. J. Olver, ed. NIST Handbook of Mathematical Functions. Cambridge University Press, 2010 (cit. on pp. 1-4, 8).

[6] R. B. Paris. "Asymptotics of the Gauss hypergeometric function with large parameters, I". In: J. Classical Anal. 2 (2013), pp. 183-203 (cit. on p. 1).

[7] R. B. Paris. "Asymptotics of the Gauss hypergeometric function with large parameters, II". In: J. Classical Anal. 3 (2013), pp. 1-15 (cit. on p. 1).

[8] G. N. Watson. "Asymptotic expansions of hypergeometric functions". In: Trans. Cambridge Philos. Soc. 22 (1918), pp. 277-308 (cit. on p. 1).

[9] John Wojdylo. "On the coeffcients that arise from Laplace's method". In: J. Comput. Appl. Math. 196 (2006), pp. 241-266 (cit. on p. 4).

[10] John Wojdylo. "Computing the Coefficients in Laplace's Method". In: SIAM Rev. 48 (2006), pp. 76-96 (cit. on p. 4).

\section{Author address}

(i) J. F. Harper, School of Mathematics and Statistics, Victoria University of Wellington, PO Box 600, Wellington 6140, NEW ZEALAND. mailto: john.harper@vuw.ac.nz 